Accepted by ApJ, February 18, 2005

Preprint typeset using $\mathrm{LAT}_{\mathrm{E}} \mathrm{X}$ style emulateapj v. 6/22/04

\title{
A SIMPLE TEST FOR THE EXISTENCE OF TWO ACCRETION MODES IN ACTIVE GALACTIC NUCLEI
}

\author{
Sebastian Jester \\ Fermilab, MS 127, PO Box 500, Batavia IL, 60510, USA \\ Accepted by ApJ, February 18, 2005
}

\begin{abstract}
By analogy to the different accretion states observed in black-hole X-ray binaries (BHXBs), it appears plausible that accretion disks in active galactic nuclei (AGN) undergo a state transition between a radiatively efficient and inefficient accretion flow. If the radiative efficiency changes at some critical accretion rate, there will be a change in the distribution of black hole masses and bolometric luminosities at the corresponding transition luminosity. To test this prediction, I consider the joint distribution of AGN black hole masses and bolometric luminosities for a sample taken from the literature. The small number of objects with low Eddington-scaled accretion rates $\dot{m}<0.01$ and black hole masses $M_{\mathrm{BH}}<10^{9} M_{\odot}$ constitutes tentative evidence for the existence of such a transition in AGN. Selection effects, in particular those associated with flux-limited samples, systematically exclude objects in particular regions of the $\left(M_{\mathrm{BH}}, L_{\mathrm{bol}}\right)$ plane. Therefore, they require particular attention in the analysis of distributions of black hole mass, bolometric luminosity, and derived quantities like the accretion rate. I suggest further observational tests of the BHXB-AGN unification scheme which are based on the jet domination of the energy output of BHXBs in the hard state, and on the possible equivalence of BHXB in the very high (or steep power-law) state showing ejections and efficiently accreting quasars and radio galaxies with powerful radio jets.
\end{abstract}

Subject headings: Galaxies: active - Galaxies: jets - Quasars: general - X-rays: binaries Accretion, accretion disks

\section{INTRODUCTION}

It has recently been shown that there is a common scaling relation between $\mathrm{X}$-ray luminosity, radio luminosity and black hole mass in black-hole X-ray binaries $(\mathrm{BHXBs})$ and active galactic nuclei (AGN), strongly suggesting that the accretion mechanism in both classes of objects is identical (Merloni et al. 2003; Falcke et al. 2004). Given this universality of accretion physics, it is reasonable to assume that the different observational states of BHXB should also exist in AGN (Meier 2001; Maccarone, Gallo, \& Fender 2003). In this paper, I suggest a simple test looking for observational consequences of such an equivalence of accretion states.

The observational states of BHXBs are distinguished by different spectral shapes and, to some extent, different luminosities. They are identified with different physical modes of accretion onto a compact object (see the review by McClintock \& Remillard 2004, and references therein; these authors suggest a non-standard state nomenclature which will be used here). One marked transition between accretion states occurs at Eddington ratios of order 1-10\%, between the "hard X-ray, steady jet" (conventionally called "low (luminosity)/hard (spectrum)") state, and the "thermal-dominant" ("high/soft") state. The difference between the hard and thermal-dominant observational states is ascribed to a difference in the state of the underlying accretion disk around a stellar-mass black hole. The thermal dominant state is identified with radiatively efficient accretion through a standard Shakura \& Sunyaev (1973) disk, while the hard state is identified with a radiatively inefficient accretion flow that replaces the innermost part of the standard disk

Electronic address: jester@fnal.gov at low accretion rates (RIAF; e.g., Esin, McClintock, \& Narayan 1997, and references therein). A second transition occurs at Eddington ratios of $20-30 \%$, where the standard Shakura \& Sunyaev (1973) thin disk becomes unstable. There, the spectrum changes from being dominated by thermal emission to showing a "steep powerlaw" (very high state), i.e., an X-ray spectrum that is harder than a pure blackbody but softer than that of the hard state. This transition may also be linked to a decrease in the radiative efficiency and hence a smaller thermal contribution to the total emission. Alternatively, an increased contribution from inverse Compton scattering in a magnetically heated corona may be responsible for the harder spectrum (Haardt \& Maraschi 1991).

The link between the state of the accretion disk and presence or absence of a jet is an important part of the picture currently emerging for BHXBs and its analogy in AGN. As implied by its name, the hard/steady-jet state is the only accretion state for which continuous, steady jets have been observed (and quite possibly are always present). When an object enters the thermal-dominant state, the radio emission associated with the steady jet is quenched (Gallo, Fender, \& Pooley 2003, e.g.). Similarly, there are suggestions that the radio galaxies and quasars with powerful jets are analogues of black-hole binaries in the steep power-law state (or making the transition to that state; see Fender, Belloni, \& Gallo 2004) which show transient X-ray and radio flares, interpreted as discrete ejections of high-velocity jets (Meier 2001; Gallo et al. 2003).

While the detailed physics of accretion flows are subject of ongoing research and debate, the generic feature of the models for the transition between the hard and thermal dominant state is a change in the radiative efficiency 
at some critical value of $\dot{m}$, the mass accretion rate $\dot{M}$ normalized to the Eddington accretion rate. This transition occurs at low accretion rates $\dot{m}=\dot{m}_{\text {crit }} \approx 0.02$. I show here that it is a consequence of such a state transition that there is a change in the distribution of accretion flow luminosities at the critical accretion rate $\dot{m}=\dot{m}_{\text {crit }}$ (§2). Using data from the literature, I examine whether the distribution of observed AGN black hole masses and luminosities is consistent with this aspect of a state transition in accretion flows (§3). The theoretical and observational picture of the second state transition, from thermal dominant to steep power law, is much less clear. Therefore, I concentrate on the low-efficiency transition in the first part of the paper. However, the existence of this accretion state may be the key to resolving some apparent contradictions of the simplest unification picture, which are discussed in $\S 4$. I conclude in $\S 5$.

\section{EFFECT OF A RADIATIVE EFFICIENCY CHANGE ON THE JOINT DISTRIBUTION OF LUMINOSITIES AND BLACK HOLE MASSES}

In this section, I show that a transition from a radiatively inefficient accretion state (a RIAF in general) to one with much higher radiative efficiency (a radiatively efficient accretion flow [REAF] such as a standard Shakura \& Sunyaev 1973 disk) is expected to change the density of objects in the $\left(M_{\mathrm{BH}}, L_{\mathrm{bol}}\right)$ plane at the luminosity corresponding to the transition accretion rate $\dot{m}_{\text {crit }}$. I assume the simplest possible generic model of an accretion state transition occurring at low radiative efficiency. The notation used here follows Esin et al. (1997), but the argument is germane to any transition from RIAF to REAF, whatever the detailed physical theory of either state may be. Thus, even if the form of the radiative efficiency is in actuality different from that used here, the method of identifying the difference between different accretion states by considering the distribution of objects in the $\left(M_{\mathrm{BH}}, L_{\mathrm{bol}}\right)$ plane is universally applicable.

The accretion flow radiates a fraction $\zeta$ of the rest mass of accreted matter, so that the radiative bolometric luminosity $L_{\text {bol }}$ and the physical accretion rate $\dot{M}$ are related by

$$
L_{\mathrm{bol}}=\zeta \dot{M} c^{2}
$$

In accretion disk models, the radiative efficiency does not depend directly on $\dot{M}$, but only on the dimensionless accretion rate $\dot{m}=\dot{M} / \dot{M}_{\text {Edd }}$ (see, e.g., Chen et al. 1995; Esin et al. 1997). In this case, using the accretionrate dependent efficiency in Equation 1 makes the definition of $\dot{M}_{\text {Edd }}$ circular, because $\dot{M}_{\text {Edd }}$ depends on the efficiency, which depends on $\dot{m}$, which depends on $\dot{M}_{\text {Edd }}$. Therefore, if the radiative efficiency varies with accretion rate, it is necessary to define $\dot{M}_{\text {Edd }}$ for a fixed fiducial value of the radiative efficiency. This is usually chosen as $\zeta_{\text {fid }}=0.1$ (Narayan \& Yi 1995; Esin et al. 1997, but compare Chen et al. 1995; Blandford \& Begelman 1999; Marchesini et al. 2004, e.g., who use $\zeta_{\text {fid }}=1 ; \dot{m}=1$ corresponds to $L_{\mathrm{bol}}=L_{\mathrm{Edd}}$ if and only if the same value is used for the efficiency in the calculation of $\dot{M}$ and $\dot{M}_{\text {Edd }}$ from $L_{\text {bol }}$ and $L_{\text {Edd }}$, respectively). $\dot{M}_{\mathrm{Edd}}$ is then given by

$$
\dot{M}_{\text {Edd }}=L_{\text {Edd }} /\left(0.1 c^{2}\right)
$$

$$
=2.2 \times 10^{-8} \frac{M_{\mathrm{BH}}}{M_{\odot}} M_{\odot} \mathrm{yr}^{-1}
$$

For all purposes other than calculating $\dot{M}_{\text {Edd }}$, I separate the conversion efficiency $\zeta$ into $\zeta=\epsilon \eta$. Here, $\eta$ is the dissipation efficiency, the fraction of the rest mass energy of accreted matter that is liberated by the accretion process. This energy may either be radiated away, swallowed by the black hole as thermal or kinetic energy, or converted into kinetic energy of a disk wind or jets (Narayan \& Yi 1995; Blandford \& Begelman 1999; Fender et al. 2003). The fraction that is radiated away is denoted by $\epsilon$. Since accreting matter has to lose the same binding energy in both presumed accretion states, I assume that universally $\eta=0.1$. The power liberated by the accretion flow is thus a constant $P=0.1 \dot{M} c^{2}$, while the radiated luminosity is given by

$$
\begin{aligned}
L_{\mathrm{bol}} & =\epsilon \dot{m} L_{\mathrm{Edd}} \\
& =\epsilon \dot{m} \frac{M_{\mathrm{BH}}}{M_{\odot}} 1.26 \times 10^{31} \mathrm{~W} .
\end{aligned}
$$

In the accretion state transition scenario, $\epsilon$ is a property of the accretion flow solution and therefore depends on $\dot{m}$ : for $\dot{m} \leq \dot{m}_{\text {crit }}, \epsilon$ is the low radiative efficiency of a RIAF, while at $\dot{m}>\dot{m}_{\text {crit }}$, it is the high efficiency of a REAF. Esin et al. (1997) found $\dot{m}_{\text {crit }}=0.08$ based on the "strong ADAF" proposal (Narayan \& Yi 1995), while Meyer, Liu, \& Meyer-Hofmeister (2000) predict $\dot{m}_{\text {crit }}=0.02$ based on their coronal evaporation model. Observations of black-hole binaries find a value close to $\dot{m}_{\text {crit }}=0.02$ (Maccarone 2003), For pure numerical convenience, we use $\dot{m}_{\text {crit }}=0.01$ here; any conclusions that would be affected by the difference between $\dot{m}_{\text {crit }}=0.01$ and $\dot{m}_{\text {crit }}=0.02$ would be on a weak footing anyway. Again ignoring physical details, I assume the following simple form for the radiative efficiency as function of accretion rate:

$$
\epsilon= \begin{cases}\epsilon^{\mathrm{lo}}=100 \dot{m} & \text { if } \dot{m} \leq \dot{m}_{\text {crit }} \\ \epsilon^{\mathrm{hi}}=1 & \text { if } \dot{m}_{\text {crit }}<\dot{m} .\end{cases}
$$

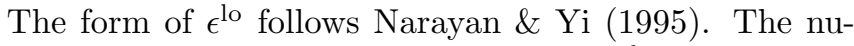
merical factor 100 in the definition of $\epsilon^{\text {lo }}$ is chosen to make the luminosity continuous as $\dot{m}$ crosses $\dot{m}_{\text {crit }}$, since transitions between the spectral states can occur without a large change in luminosity. As mentioned in the introduction, the accretion rate $\dot{m}$ should in principle be restricted to $\dot{m}<0.3$ since the standard Shakura \& Sunyaev (1973) thin disk becomes unstable and BHXBs are known to enter the steep power-law state at higher accretion rates. Lacking accurate knowledge of the radiative efficiency of that state, it appears more appropriate to concentrate on the detectability of the low-accretion rate transition, which should not be influenced by the presence of this third accretion state at high accretion rates (see $\S 3.4$ ).

The assumed prescription for the radiative efficiency in Equation 4 ignores the finding that there is a hysteresis in the transition luminosity in at least five soft X-ray transient binary systems, in the sense that the transition from the hard to the thermal dominant state occurs at a luminosity that is higher than a factor of about five than the reverse transition (see Maccarone \& Coppi 2003 and references therein; Barret \& Olive 2002). I will discuss the possible impact of this simplification in $§ 3.3$. 
It is immediately obvious from equations 3 and 4 that for fixed black hole mass, the distribution of bolometric luminosities must show the same transition to a different scaling with $\dot{m}$ at $\dot{m}=\dot{m}_{\text {crit }}$ as $\epsilon$. Thus, this transition should be detectable when considering the distribution of accreting systems in the $\left(M_{\mathrm{BH}}, L_{\mathrm{bol}}\right)$ plane, as long as the distribution of accretion rates $\dot{m}$ does not similarly change precisely at $\dot{m}_{\text {crit }}$. This assumption is consistent with the common view that $\dot{m}$ is an object-specific input parameter fixed by the surroundings of the particular accreting system (e.g., the availability of gas in the AGN host galaxy), while $\dot{m}_{\text {crit }}$ is a universal property of the accretion flow solution (although it is possible that the mass supply rate at large radii does not in fact govern the accretion rate onto the black hole; see Proga 2005, e.g.). Thus, I consider whether there any observational evidence for such a change in the distribution of observed AGN luminosities at fixed black hole mass.

\section{OBSERVATIONS}

\subsection{Results}

Figure 1 shows the distribution of bolometric luminosities and black hole masses for the sample of objects from the compilation in Woo \& Urry (2002), who list black hole masses obtained by a variety of methods, and from McLure \& Dunlop (2004), who determine virial black hole masses for objects from the Sloan Digital Sky Survey (SDSS) quasar catalog (Schneider et al. 2003) using the virial method and the correlation between AGN luminosity and size of the broad-line region (BLR). The SDSS spectroscopic quasar survey is an optically flux-limited survey of quasar candidates selected either from 5-color photometry or as optical point-source counterparts of sources detected in the FIRST radio survey (details of the selection are described in Richards et al. 2002). The objects whose mass and luminosity are taken from Woo \& Urry (2002) are taken from a variety of samples and sources. Most of the sources with black hole masses from the BLR size-luminosity relation are radio-selected quasars, while most other AGN are Seyferts in nearby galaxies, or optically selected quasars from the Bright Quasar Survey (PG objects).

The diagonal lines in Fig. 1 indicate the relation between luminosity and black hole mass for the indicated Eddington-scaled accretion rates $\dot{m}$ (equally spaced in $\log \dot{m})$ and the resulting radiative efficiency from Equation 4 . As argued above, the distribution of objects in $\dot{m}$ is not expected to change at $\dot{m}=\dot{m}_{\text {crit }}$. Therefore, the change in radiative efficiency at $\dot{m}=\dot{m}_{\text {crit }}$ should lead to a change in the density of objects in the $\left(M_{\mathrm{BH}}, L_{\mathrm{bol}}\right)$ plane at the line $\dot{m}=\dot{m}_{\text {crit }}$. In Fig. 1, the density of objects does indeed change abruptly at this line. In fact, nearly all objects from the samples used here lie above this line in the radiatively efficient regime, while only a few objects have lower $\dot{m}$ as inferred from their black hole mass, luminosity, and the radiative efficiency prescribed by Equation 4.

Figure 2 shows the equivalent of Fig. 1 for the sample of radio-loud objects from Marchesini, Celotti, \& Ferrarese (2004). That sample includes only objects with detected host galaxies and HST imaging, allowing the authors to measure the black hole mass from the correlation between host galaxy luminosity and black hole mass, as well

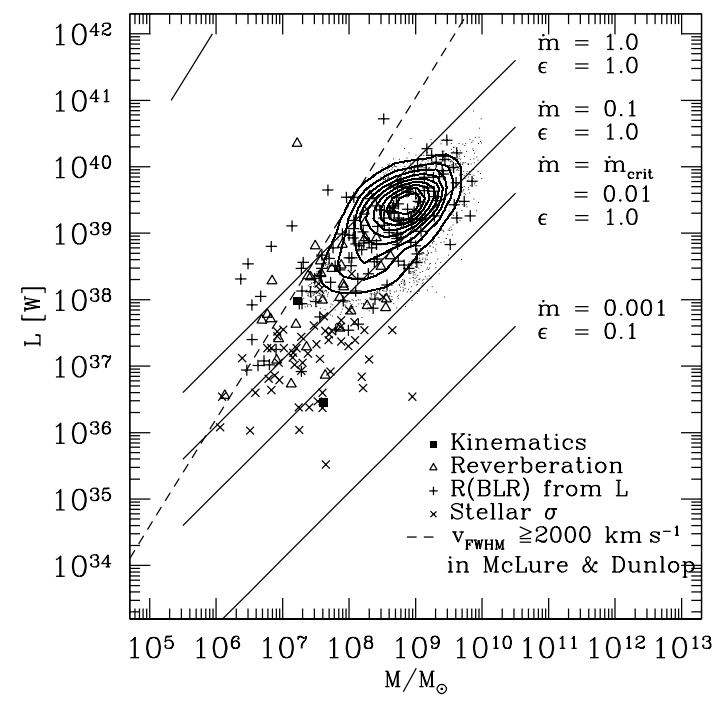

FIG. 1.- Distribution of AGN bolometric luminosities and black hole masses (the symbol type indicates the method of black hole mass determination as detailed below). The diagonal solid lines are lines of constant Eddington ratio $L_{\mathrm{bol}} / L_{\mathrm{Edd}}$. They show the bolometric luminosity as function of black hole mass from Equations 3 and 4 for the given values of the accretion rate $\dot{m}$. The radiative efficiency $\epsilon$ has a discontinuity at $\dot{m}=\dot{m}_{\text {crit }}=0.01$ which separates radiatively inefficient flows (RIAF, $\epsilon=100 \dot{m}$ ) at smaller $\dot{m}$ from radiatively efficient flows (REAF, $\epsilon=1)$ at larger $\dot{m}$. The diagonal lines are equally spaced in $\dot{m}$, so that the change in line spacing with respect to luminosity should be reflected in a change in the density of objects in the $\left(M_{\mathrm{BH}}, L_{\mathrm{bol}}\right)$ plane at the line $\dot{m}=\dot{m}_{\text {crit }}$. Contours and dots are measurements for the 12245 objects from the SDSS DR1 quasar catalog (Schneider et al. 2003), determined by McLure \& Dunlop (2004) using the relation between optical luminosity and size of the broad-line region (BLR) together with the line width of the $\mathrm{H} \beta$ or Mg II lines as virial mass indicators. These authors compute bolometric luminosities using a fixed bolometric correction for the optical luminosity. There are 12245 objects in total; the contours indicate the density of objects in bins of 0.15 dex in both axes, starting at 1 object per bin and increasing by a factor of $\sqrt{2}$ per contour. The dashed line indicates the FWHM cut $\geq 2000 \mathrm{~km} \mathrm{~s}^{-1}$ applied by McLure \& Dunlop which excludes objects above and to the left of this line. The solid line in the upper left-hand corner shows the correlated change in $L_{\mathrm{bol}}$ and $M_{\mathrm{BH}}$ that an error in an object's optical luminosity of 1 dex would produce. Other symbols are those black hole mass measurements from Woo \& Urry (2002) for which the authors have determined the bolometric luminosity. The method of black hole mass determination is indicated by the symbol type: solid squares for resolved stellar kinematics (2 objects); open triangles for reverberation mapping (36); plus signs for BLR size from optical luminosity as in McLure \& Dunlop (139); and crosses for direct measurements of the stellar velocity dispersion $\sigma$ and the $M_{\mathrm{BH}^{-}} \sigma$ relation (57).

as the nuclear optical luminosity to determine the nuclear bolometric luminosity using a bolometric correction factor. I have omitted sources with only an upper limit to the nuclear luminosity. Some of the objects classified as radio-loud quasars are also included in the sample shown in Figure 1. The accretion rate histogram derived from this figure (essentially, by a projection of this figure on a line perpendicular to the lines $\dot{m}=$ const), Marchesini et al. (2004) shows a clear bimodality in the accretion rate distribution of AGN. This bimodality is taken as strong evidence for the presence of a transition between two accretion states. The presence of a "gap" in the distribution, roughly located at $3 \times 10^{-4}<\dot{m}<10^{-2}$, requires a discontinuous change of some property of the 


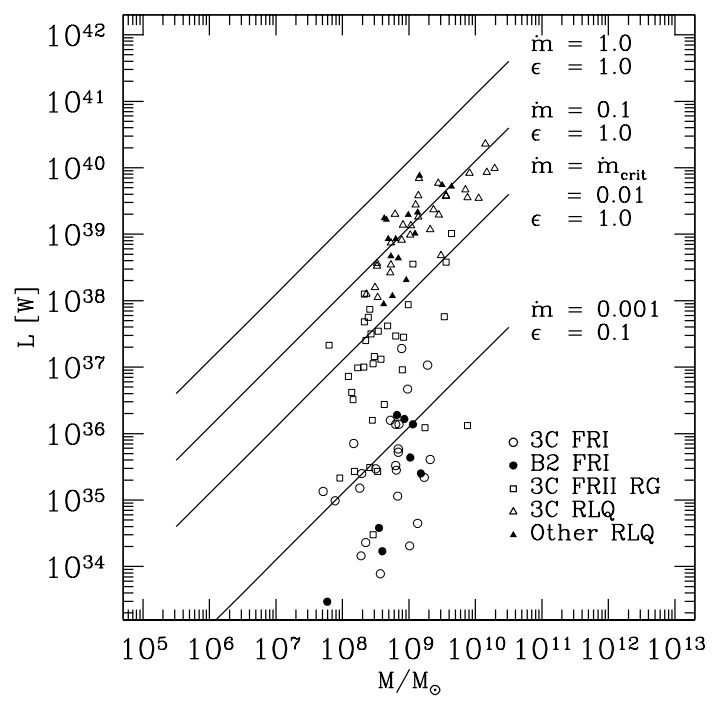

FIG. 2.- Bolometric luminosity against black hole mass for sources listed by Marchesini et al. (2004), including only sources with a measurement of the bolometric luminosity (excluding upper limits). The lines for different Eddington-scaled accretion rates are identical to those in Figure 1. Triangles indicate radio-loud quasars (empty triangles for 3CR objects, filled for objects from McLure \& Dunlop 2001 and Hutchings et al. 2002), squares are FR II radio galaxies from the $3 \mathrm{C}$ sample, and circles are low-luminosity (mostly FR I) radio galaxies from the $3 \mathrm{CR}$ (empty) and B2 (filled) samples.

accretion flow (note that in contrast to the treatment here, Marchesini et al. 2004 define $\dot{m} \equiv L_{\text {bol }} / L_{\text {Edd }}$, i.e., they use a constant radiative efficiency to avoid having to make assumptions about the radiative efficiency as function of accretion rate). Even though the accretion rate histogram clearly shows a bimodality, there is no obvious structure to the distribution of the same objects in the $\left(M_{\mathrm{BH}}, L_{\mathrm{bol}}\right)$ plane. In fact, it appears preferable to consider the full 2-dimensional distribution rather than its 1-dimensional projection, the accretion rate histogram, because the impact of selection effects is more obvious in the 2-dimensional diagram. The impact of selection effects is discussed in detail in $§ 3.5$ below.

The bulk of the 12245 objects from McLure \& Dunlop (2004) lies in the region between $\dot{m}=0.1$ and $\dot{m}=1$ (contours in Fig. 1). These are so far from the line $\dot{m}=\dot{m}_{\text {crit }}$ that these objects genuinely cannot be accreting in the low- $\dot{m}$ radiatively inefficient mode. This suggestion seems to be supported by the fact that the vast majority of SDSS quasars has a blue bump (the average SDSS quasar spectrum clearly shows the big blue bump; see Vanden Berk et al. 2001; Yip et al. 2004b). However, the implied $\dot{m}$ of many of these objects is so high that the Shakura \& Sunyaev (1973) standard disk solution does not hold any more and they should be in the third accretion state. We return to this point in the discussion section below ( $\$ 4.3)$. The lack of inefficiently accreting objects in the SDSS sample is predominantly due to the combined effect of the luminosity and flux limit for objects in the SDSS quasar catalog, from which this sample is drawn (see $\S 3.5$ below). The lack of such objects therefore does not allow to make any statement about the presence or absence of two accretion modes in AGN.
The objects from Woo \& Urry (2002) include many Seyfert galaxies with luminosities that are much lower than the limit for inclusion in the SDSS quasar catalog, in particular among the objects with $M_{\mathrm{BH}}$ from stellar velocity dispersions (crosses in Fig. 1) and those with reverberation-mapping masses (triangles). These objects are preferentially found above the line $\dot{m}=\dot{m}_{\text {crit }}=0.01$. The lack of objects with $\dot{m}<\dot{m}_{\text {crit }}$ at $M_{\mathrm{BH}}>10^{9} M_{\odot}$ again is mainly due to selection effects. But even at $M_{\mathrm{BH}}<10^{9} M_{\odot}$, there are fewer objects with $\dot{m}<\dot{m}_{\text {crit }}$ compared to $\dot{m}>\dot{m}_{\text {crit }}$ (12 below $\dot{m}_{\text {crit }}$ and 185 above). The asymmetry is perhaps most significant among the objects with masses from host galaxy stellar velocity dispersions, which are more likely to include low-luminosity AGN: only 11 of these are below $\dot{m}_{\text {crit }}$, compared to 46 above. The significance of this difference is hard to quantify because the objects listed by Woo \& Urry (2002) by construction do not constitute a complete sample. But the small number of objects with $\dot{m}<\dot{m}_{\text {crit }}$ at $M_{\mathrm{BH}}<10^{9} M_{\odot}$ may be taken at least as weak suggestive evidence for the presence of a radiatively inefficient accretion mode.

In stark contrast to the small number of low-efficiency objects in Fig. 1, the Marchesini et al. (2004) sample shown in Fig. 2 shows a substantial number of objects in the low-efficiency regime. The key difference is that Marchesini et al. (2004) used only optical observations and a bolometric correction to determine bolometric luminosities for these low-power radio galaxies, while Woo \& Urry (2002) explicitly excluded radio galaxies in their determinations of bolometric luminosities because "obscuration and beaming are significant" in these sources. By contrast, Marchesini et al. (2004) argue (based on the analysis by Chiaberge et al. 2002) that nuclear obscuration is not important in these low-power radio galaxies, so that the nuclear optical luminosity can be used to infer the bolometric luminosity. This finding is disputed by Cao \& Rawlings (2004) - while they agree with Chiaberge et al. (2002) that the optical core flux of these objects is dominated by jet emission, they conclude that the jet emission comes from scales larger than the obscuring material. Thus, I refrain from drawing any conclusion from the large number of low-luminosity (and hence low-accretion rate) sources in Figure 2, since they would hinge on which assumptions are adopted about the nature of the nuclei of low-luminosity radio galaxies.

I next consider the impact of the other assumptions going into construction of the distributions in the $\left(M_{\mathrm{BH}}, L_{\mathrm{bol}}\right)$ plane on the results obtained here.

\subsection{Impact of assumptions}

In order to assess the significance of any conclusions about the presence or absence of two accretion modes, it is necessary to consider the impact of the assumptions going into the determination of the distribution of objects in the $\left(M_{\mathrm{BH}}, L_{\mathrm{bol}}\right)$ plane. Perhaps the most serious caveat has already been mentioned above: the accretion rate $\dot{m}$ might not necessarily be the same as the mass supply rate, but could be set by processes internal to the accretion disk (Proga 2005), so that the value of $\dot{m}$ may be a property of the accretion disk solution rather than an independent parameter set by the availability of gas supply in the host galaxy, as has been assumed here. If this is the case, the distribution of objects in the 
$\left(M_{\mathrm{BH}}, L_{\mathrm{bol}}\right)$ plane would still provide information about the physics of accretion disks if the mass supply rate could be determined independently.

A detailed discussion in $\S A .1$ of the appendix shows that errors in the determination of black hole mass and bolometric luminosities would either blur the transition region or systematically shear the distribution of points in the $\left(M_{\mathrm{BH}}, L_{\mathrm{bol}}\right)$ plane, making the detection of the transition more difficult, but not impossible. In $\S \mathrm{A} .2$ of the appendix, I conclude that the bolometric luminosity constitutes the most reliable measure of accretion luminosity. However, large systematic errors are introduced in bolometric luminosities by deriving bolometric corrections from one sample (the objects in the library of AGN SEDs by Elvis et al. 1994) but applying them to the SDSS quasar sample, which goes beyond traditional UV excess selection and includes objects with much redder optical colors, and therefore possibly different SEDs in other wavelength regions, than the samples on which the bolometric corrections are based.

In the remainder of this section, I consider in detail the impact of assumptions about the values for critical accretion rate and radiative efficiency, and of the sample selection function on this distribution. I will then discuss the implications of the observed distributions.

\subsection{Values for critical accretion rate and radiative efficiencies}

In order to determine the accretion rate $\dot{m}$ from the observable $L_{\text {bol }}$, it was necessary to assume a value for the fraction of the accreted matter's rest-mass energy that is dissipated by the accretion process, and to assume radiative efficiencies for the different accretion states. In addition, the location of the line dividing efficient from inefficient accretors in the $\left(M_{\mathrm{BH}}, L_{\mathrm{bol}}\right)$ plane obviously depends on the value of $\dot{m}_{\text {crit }}$.

Here, the fraction of rest-mass energy that is liberated as accretion power was taken to be $\eta=0.1$ universally. However, this value in fact depends on the location of the last stable orbit around the black hole, which in turn depends the black hole spin. For a non-rotating black hole (Schwarzschild metric), $\eta=0.06$. The efficiency of accretion on a rotating black hole (Kerr metric) can be lower than the Schwarzschild value if it is counterrotating with respect to the disk, while a maximally corotating black hole would result in $\eta=0.42$. As there is likely a large variation of the black hole spin from AGN to AGN based on its particular accretion history, the value of $\eta$ will also differ from object to object. The value of $\eta$ changes the location of the lines $\dot{m}=$ const. in Fig. 1, i.e., there would be a different set of these lines for each value of $\eta$. The appropriate simplification would be to use the ensemble average of $\eta$ to draw this set of lines; since an accurate measurement of $\eta$ is not available for any object, use of the conventional value $\eta=0.1$ appears most appropriate.

I have chosen $\dot{m}_{\text {crit }}, \epsilon^{\mathrm{lo}}$, and $\epsilon^{\text {hi }}$ to match observations of black-hole binaries approximately. If the true values of $\dot{m}_{\text {crit }}, \epsilon^{\text {lo }}$, and $\epsilon^{\text {hi }}$ were smaller than assumed here, the transition between efficient and inefficient accretion would occur at smaller luminosities, where there are not many objects in Fig. 1. In this case, all objects in Fig. 1 might in fact be in the efficient state, and the luminosity range occupied by inefficiently accreting objects has not yet been reached.

Another complication arises from the fact that there is be a hysteresis loop between the two accretion states in black-hole binaries, with the low-high transition occurring at a different accretion rate than the high-low transition (Maccarone \& Coppi 2003, and references therein). These authors find that the transition back to the lowefficiency hard state occurs at a luminosity $\sim 5$ times lower than the hard-to-soft transition from low to high efficiency, so that objects with identical luminosity can have different accretion rates. If AGN can undergo an accretion state change during their active phase, this would apply to them, too, so that the instantaneous luminosity would not reveal the instantaneous accretion rate. The adopted value of $\dot{m}_{\text {crit }}$ would again need to be interpreted as an ensemble average.

In all these cases, the transition between efficient and inefficient accretion would still be visible in the $\left(M_{\mathrm{BH}}, L_{\mathrm{bol}}\right)$ plane, but the transition would be blurred by the scatter of true efficiencies about the assumed average value, or the location of the transition would be moved. In this case, it might occur in a region that currently does not contain any objects. These difficulties can therefore be overcome with observations of more objects.

\subsection{Ignoring a possible third accretion state}

Even if the parameters chosen for the low-accretion rate transition are correct, there is a third superEddington accretion mode with a different efficiency of the standard thin disk. In fact, the standard thin-disk model becomes self-inconsistent and must be modified to include advection above $\dot{m}=0.2-0.3$ (Koratkar \& Blaes 1999, e.g.), i.e., they become radiatively inefficient, too. Accretion at even higher rates could become even more radiatively inefficient (see Begelman \& Meier 1982; Abramowicz 2004, e.g.). "Super-Eddington" would then refer to the fact that the accretion rate exceeds the Eddington rate for the fiducial efficiency, while the radiative luminosity may still be below the Eddington luminosity because of the decrease in the actual radiative efficiency. Consideration of this accretion state is particularly relevant since BHXBs enter a steep power-law state at roughly $30 \%$ of the Eddington luminosity, with a harder X-ray spectrum than the thermal-dominated state.

The physics of these high-accretion rate inefficient disks are the subject of ongoing research. No universally accepted predictions are available yet for their radiative efficiency and possible time-dependent behavior. Generically, though, the radiative efficiency must be dropping with increasing $\dot{m}$ just above this second critical accretion rate. The accretion luminosity would therefore increase more slowly than $\dot{m}$. It could even decrease with increasing $\dot{m}$ if the accretion efficiency scales like $\dot{m}^{p}$ with $p<-1$ in the transition region. The same radiative luminosity may then be produced either by a standard accretion flow with modest $\dot{m}$ and high $\epsilon$, or by a superEddington flow with high $\dot{m}$ and modest $\epsilon$. In any case, a second transition to a low-efficiency state would lead to a "pileup" of sources in the $\left(M_{\mathrm{BH}}, L_{\mathrm{bol}}\right)$ plane near the super-Eddington $\dot{m}_{\text {crit }}$. In this context, it may be of relevance that the contours for the SDSS quasars in Fig. 1 are peaked around the line $\dot{m}=0.3$. 


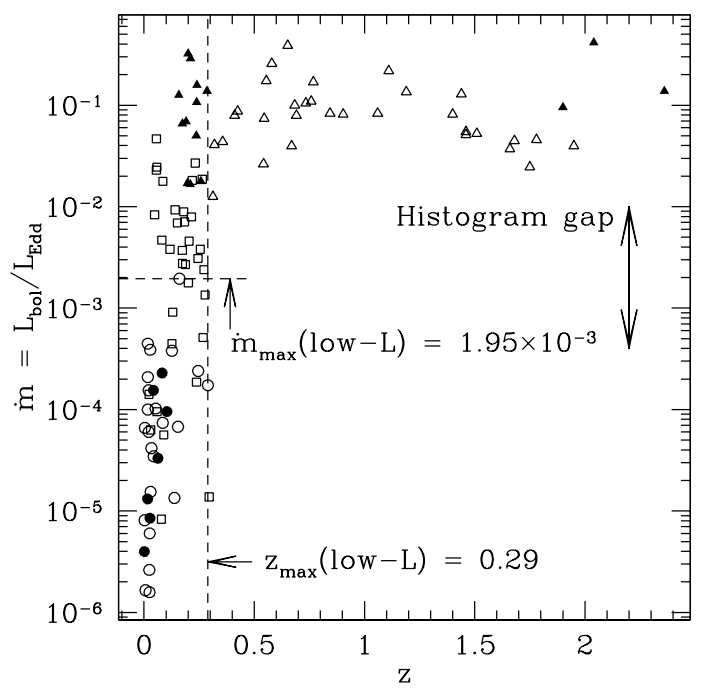

FIG. 3.- Dimensionless accretion rate $\dot{m}$ against redshift $z$ for the Marchesini et al. (2004) objects shown in Figure 2; for consistency with that work, this figure defines $\dot{m} \equiv L_{\mathrm{bol}} / L_{\mathrm{Edd}}$, i.e., with a constant radiative efficiency. Symbols as in Figure 2: triangles for radio-loud quasars, empty triangles for $3 \mathrm{CR}$ and filled from two other samples; squares for 3CR FR II radio galaxies; circles for lowluminosity radio galaxies from 3CR (empty) and B2 (filled). The double arrow indicates the location of the gap identified by Marchesini et al. (2004) in the accretion rate histogram. The dashed lines show the maximum value of $\dot{m}$ and $z$ for the set of low-luminosity radio galaxies (open circles). These constitute the majority of low$\dot{m}$ objects, but are drawn from a completely different volume than the $3 \mathrm{CR}$ radio-loud quasars (open triangles), which constitute the majority of high- $\dot{m}$ objects: the former are limited to $z<0.29$ and $\dot{m} \leq 1.95 \times 10^{-3}$, while the latter are found at $z \geq 0.3$ and $\dot{m}>10^{-2}$. Figure 4 below compares the accretion rate histograms of the full sample, and of a revised sample which includes only objects at $z \leq 0.29$.

In an extreme case, the radiative efficiency of the super-Eddington state could be so low that the luminosity of objects with any $M_{\mathrm{BH}}$ can be produced in a radiatively inefficient flow. In this case, there may in fact be no standard radiatively efficient accretion flows at all (cf. Chen et al. 1995). Unless this is the case, this high-Eddington rate transition at $\dot{m}=0.2-0.3$ should not influence the detectability of the low-Eddington rate transition at $\dot{m}_{\text {crit }} \approx 0.01$.

\subsection{Impact of sample selection effects}

The selection of a sample of AGN for which black hole masses and absolute luminosities are to be determined always restricts the resulting distribution of objects to certain regions of the $\left(M_{\mathrm{BH}}, L_{\mathrm{bol}}\right)$ plane. This can happen both explicitly, by a luminosity or line width cut, e.g., or implicitly, because the distribution of AGN luminosities and black hole masses over redshift systematically removes objects from flux-limited surveys, e.g. The correct interpretation of the observed distribution of objects in the $\left(M_{\mathrm{BH}}, L_{\mathrm{bol}}\right)$ plane requires to take into account these selection effects.

\subsubsection{Selection effects in Marchesini et al. (2004) sample}

The selection effects of the Marchesini et al. (2004) sample of objects are the simplest, as they use mostly

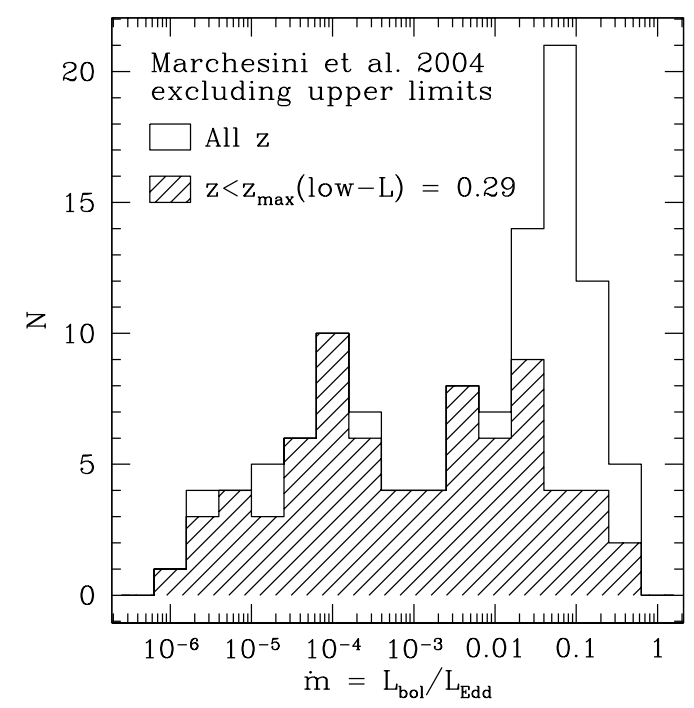

FIG. 4.- Accretion rate histogram for Marchesini et al. (2004) objects, excluding objects with upper limits on the bolometric luminosity. The open histogram shows the histogram of $\dot{m}$ for the entire remaining sample, while the shaded histogram shows the histogram for objects at $z \leq 0.29$, which is the highest redshift of any source with $\dot{m} \leqslant 2 \times 10^{-3}$. Thus, the shaded histogram excludes those sources located in the volume in which low- $\dot{m}$ sources are inaccessible to observations. This "equal-redshift-range" sample does not show a pronounced gap in the accretion rate distribution any more.

objects from two complete flux-limited radio surveys, the 3CR and B2 surveys. The 3CR (Laing et al. 1983; Spinrad et al. 1985) is a $178 \mathrm{MHz}$ survey of 13,920 square degrees of sky to $10.9 \mathrm{Jy}$. It includes a mix of lowpower FRI (radio luminosity at $178 \mathrm{MHz}$ in the range $\left.10^{24}<L_{178}<10^{28} \mathrm{~W} \mathrm{~Hz}^{-1}\right)$ and higher-power FRII radio galaxies $\left(10^{25}<L_{178}<10^{28} \mathrm{~W} \mathrm{~Hz}^{-1}\right)$ at $z \leq 0.3$, as well as radio-loud quasars at $z \geq 0.3$, with luminosities in the range $10^{27}<L_{178}<10^{29} \mathrm{~W} \mathrm{~Hz}^{-1}$. The B2 survey (Colla et al. 1975) is a survey of about onehalf the area of the $3 \mathrm{C}$ with a flux limit of $0.2-0.25 \mathrm{Jy}$ and a magnitude limit $m_{V}<16.5$ on the galaxies identified optically with the radio sources, i.e., it samples a smaller volume than the $3 \mathrm{C}$. Therefore, it predominantly includes low-luminosity objects (high-luminosity objects have a lower volume density and therefore require a large-volume sample to be found): the B2 objects used by Marchesini et al. (2004) are all at $z \leq 0.15$ and have $10^{22}<L_{178}<10^{26} \mathrm{~W} \mathrm{~Hz}^{-1}$. The sample is augmented by further radio-loud quasars in the redshift range $0.15 \leq z \leq 0.3$. Thus, the total sample has substantial overlap in $L_{178}$ and redshift. However, Figure 3 shows that the majority of high-accretion rate objects are drawn from a completely different volume than all the low-accretion rate objects. Low-accretion rate objects, in particular the low-luminosity radio galaxies from the B2 sample, are exclusively found at $z \leq 0.29$, while all of the $3 \mathrm{C}$ radio-loud quasars are at $z>0.3$. The only high-accretion rate objects at $z<0.3$ included in the Marchesini et al. (2004) histogram are the $3 \mathrm{C}$ radio galaxies (i.e., objects with both powerful radio jets as well as broad emission lines and some non-stellar continuum, but with lower optical luminosities than radio-loud quasars) as well as the radio-loud quasars from McLure 
\& Dunlop (2001).

Figure 4 shows the effect of removing all sources at $z>0.3$ from the sample, where objects with $\dot{m} \lesssim 10^{-2}$ are inaccessible to the surveys used. The high- $\dot{m}$ peak in the accretion rate histogram disappears nearly completely, and moves from $\dot{m}_{\text {peak }} \approx 0.1$ to $\dot{m}_{\text {peak }} \approx 0.01$. There is no clear bimodality any more. Thus, not only is the gap in the accretion rate distribution not obvious when considering the full 2-dimensional distribution of objects from Marchesini et al. (2004) in the $\left(M_{\mathrm{BH}}, L_{\mathrm{bol}}\right)$ plane (Fig. 2), but the gap is an artifact of selection effects: high- $\dot{m}$ objects are predominantly found at $z \gtrsim 0.3$ in the samples used, while low- $\dot{m}$ objects are restricted to $z \lesssim 0.3$. Furthermore, even after applying an identical redshift cut to the samples used by Marchesini et al. (2004), the volumes sampled to find the different subsets of objects are still different because of the different areas of sky and that have been surveyed by the B2 and the $3 \mathrm{C}$ survey, and because of the optical flux limit imposed on B2 galaxy identifications.

\subsubsection{Selection effects in the SDSS quasar sample}

The same criticism (objects with different accretion rates being drawn from different volumes) also applies, of course, to the objects shown in Figure 1. The objects for which McLure \& Dunlop (2004) determine black hole masses are drawn from the SDSS DR1 quasar catalog (Schneider et al. 2003). This catalog is restricted in the $i$-band, both by flux limit and by a limit in absolute magnitude $M_{i} \leq-22$, which roughly corresponds to $L_{\text {bol }} \geq 10^{38} \mathrm{~W}$ (using the relation between $L_{\text {bol }}$ and $M_{B}$ given by McLure \& Dunlop 2004 and approximating quasar spectra as a power law with $f_{\nu} \propto \nu^{-0.5}$, resulting in $\left.M_{B}-M_{i}=0.3\right)$. This luminosity cut means that objects with $\dot{m} \leq \dot{m}_{\text {crit }}=0.01$ are only included in this quasar catalog if they satisfy $M_{\mathrm{BH}} \gtrsim 10^{9} M_{\odot}$. The flux limit of the quasar survey restricts the detection of such objects to $z \lesssim 0.33$ for ugri-selected objects $\left(m_{i} \leq 19.1\right)$ and $z \lesssim 0.53$ for griz-selected objects $\left(m_{i} \leq 20.2\right)$. The surveyed volume is therefore limited, and hence the number of such objects that can be found by the SDSS.

At higher redshifts, the surveyed volume is much larger, but now the flux limit introduces a redshiftdependent luminosity limit. As discussed by McLure \& Dunlop (2004), the use of the luminosity in the black hole estimation in a flux-limited implies a correlation between black hole mass and redshift, leading to an effective lower limit on the black hole mass as a function of redshift. Thus, the SDSS quasar survey misses high-mass black holes with low luminosities. Furthermore, McLure \& Dunlop (2004) include only objects with $v_{\text {FWHM }} \geq 2000 \mathrm{~km} \mathrm{~s}^{-1}$ in their sample, which excludes objects to the left and above the dashed line. This cut is equivalent to a mass-dependent upper limit on $\dot{m}$ for objects in the sample.

In general, the volume density of faint AGN (more than 3 magnitudes fainter than those observed by the SDSS) increases towards redshifts around 2 in the same way as that of luminous quasars (Wolf et al. 2003), i.e., faint AGN outnumber bright ones at all redshifts. We are therefore missing black hole mass determinations for a substantial fraction of the AGN population, with a strong bias against low-luminosity AGN at high redshifts.

\subsubsection{Selection effects for remaining sources}

The remainder of the objects have unquantifiable selection effects, since they are not drawn from samples with well-defined selection criteria. However, Woo \& Urry (2002) give a detailed discussion of possible selection effects in the construction of a diagram such as Fig. 1. The important incompleteness for the present discussion is again the lack of objects with high black hole masses $\left(M_{\mathrm{BH}} \geq 10^{8} M_{\odot}\right)$ and low luminosities $\left(L_{\mathrm{bol}} \leq 10^{38} \mathrm{~W}\right)$. Since black hole mass correlates with the bulge luminosity of the host galaxy (Marconi \& Hunt 2003, and references therein), a fraction of these objects is missing from AGN samples at all redshifts: a low-luminosity AGN is more difficult to detect against the more luminous host galaxy, and the small volume of the low-redshift universe means that high-mass black holes are rare in it. There is an additional observer bias towards high-luminosity AGN: black hole masses are typically first determined for objects drawn from the brightest samples, such as the 3C radio survey, the Bright Quasar Survey, or the SDSS quasar survey, even though faint AGN are more numerous.

Furthermore, there is some natural practical bias towards a certain type of AGN for a certain method of black hole mass determination. For example, the use of the stellar velocity dispersion method is only possible for AGN which are sufficiently nearby and of low luminosity to allow observation of the host galaxy. A sample that exclusively uses this method will therefore be biased against high-luminosity AGN with low-mass black holes, because these reside in low-luminosity hosts which are likely to be outshone by the nucleus. Selection effects of this kind can be avoided by making use of the broadest possible range of black hole mass determination methods.

In conclusion, the sample selection function severely influences the observed distribution of objects in all regions of the $\left(M_{\mathrm{BH}}, L_{\mathrm{bol}}\right)$ plane. In particular, flux limits on AGN surveys (both explicit flux limits in complete surveys, and implicit flux limits because the faintest AGN are practically unobservable) bias any survey against the inclusion of low-luminosity objects, whose black hole masses are crucial in detecting the expected difference between efficiently and inefficiently accreting objects. Finally, there is a population of obscured AGN that might be at least as numerous as known AGN populations (Risaliti \& Elvis 2004; Treister et al. 2004), but not many black hole mass measurements exist for Type 2 AGN, and none exist for obscured sources at high redshift.

Thus, considering distributions like those in Figure 1 may yield a misleading picture because every data point obtains an equal weight, regardless of the volume that had to be surveyed to find it, and regardless of the properties of objects that are excluded by selection effects. The effect of removing sources at high redshift, where low-luminosity AGN are not included in flux-limited samples, from Fig. 1 would be similar to the effect on the accretion rate histogram in that it removes the bulk of objects which are clearly at $\dot{m}>0.01$. The more sound way to perform the test suggested here will be to determine the volume density of objects with a given $L_{\mathrm{bol}}$ and $M_{\mathrm{BH}}$, preferably in a volume-limited sample.

However, the evidence cited above in favor of the ex- 
istence of a transition relied mostly on the asymmetry in the $\dot{m}$ distribution of objects with masses from host galaxy stellar velocity dispersions. Since these are more likely to include low-luminosity AGN than flux-limited quasar surveys, this conclusion is not affected severely by the selection effects discussed in this subsection.

\section{DISCUSSION}

The main aim of this paper is to point out that a transition in the radiative efficiency at some critical accretion rate $\dot{m}_{\text {crit }}$ should lead to a change in the distribution of AGN in the $\left(M_{\mathrm{BH}}, L_{\mathrm{bol}}\right)$ plane, and that this prediction is testable with presently available techniques. If the analogy between AGN and black-hole binaries is complete, the difference between the different accretion states should not only be evident in the $\left(M_{\mathrm{BH}}, L_{\mathrm{bol}}\right)$ plane, but also manifest itself in a spectral difference between efficiently and inefficiently accreting objects. This section considers the spectral evidence for an analogy of AGN and black-hole binary accretion states, first for the 12 low- $\dot{m}$ objects identifiable in Fig. 1 and then for the remaining objects which are presumably in a highefficiency accretion state. I also discuss whether the spectral and jet properties of AGN in general fit into the same classification scheme as black-hole binaries. Before considering these points, I briefly discuss the evidence for the presence of a transition in the accretion properties of radio-loud AGN presented by Marchesini et al. (2004).

\subsection{A bimodality in the $\dot{m}$ distribution of $A G N$ with powerful jets?}

In $§ 3.5 .1$ above, I showed that the obvious bimodality in the accretion rate histogram of radio-loud AGN presented by Marchesini et al. (2004) is to a large degree a consequence of sample selection effects, with high- $\dot{m}$ objects (high-efficiency accretors) being drawn from a much larger volume than those with low $\dot{m}$ (low-efficiency accretors). This does not, however, imply that the conclusions drawn by Marchesini et al. (2004) are necessarily invalid - §3.5.1 merely shows that selection effects are responsible at least in part for the apparent bimodality, but not that there is no such bimodality, even though Figure 2 shows no obvious gap in the distribution of radio-loud objects in the $\left(M_{\mathrm{BH}}, L_{\mathrm{bol}}\right)$ plane. To clarify this issue, a sample of AGN is needed which includes all objects with $\dot{m}$ in the range under consideration.

\subsection{Spectral evidence for inefficient accretion?}

We concluded above that the small number of objects in the sample of AGN shown in Figure 1 with $\dot{m}<\dot{m}_{\text {crit }}$ at $M_{\mathrm{BH}}<10^{9} M_{\odot}$ may be taken as weak suggestive evidence for the presence of a radiatively inefficient accretion mode. The 12 objects which have $M_{\mathrm{BH}}<10^{9} M_{\odot}$ and $\dot{m}<\dot{m}_{\text {crit }}=0.01$ are Mrk 3 and 270, and NGC 513, $1052,2110,2841,3786,3998,4258,4339,5929$, and 6104 ; NGC 4258 has a direct black hole mass measurement from water maser kinematics, while the black hole masses for the remainder have been determined from the stellar velocity dispersion of the host galaxy and the $M-\sigma$ relation. Is there any spectral evidence that these are in a low-efficiency accretion state? If the black-hole binary scheme applied in complete analogy to AGN, the lowefficiency accretion state ought to show a steady jet, and they should not be dominated by thermal emission from the accretion disk, i.e., they should not have a "big blue bump".

These objects are all low-luminosity AGN. Most are classified as Seyfert galaxies (including both Seyfert 1, 2 , and intermediate types) or low-ionization nuclear emission-line region (LINER; see Heckman 1980, e.g.). Some of the narrow-line objects show evidence for hidden broad-line regions in polarized light. Mrk 3 and NGC 1052, 2110, and 4258 show clear radio jets (Liu \& Zhang 2002), while the evidence for a radio jet in NGC 2841 is only tentative so far (Nagar et al. 2002). Thus, about one third of the objects have clear detections of radio jets. However, the lack of clear jet detections in the remainder of the objects does not necessarily imply the absence of jets, but may be due to the lack of suitable observations. Indeed, among the hard-state galactic BHXBs, only Cygnus X-1 has an imaged jet, while the presence of jets in other objects is inferred by indirect arguments, typically lower limits on the source size inferred from the radio brightness temperature.

Ho (1999) considers the SEDs of seven low-luminosity AGN, showing that the "big blue bump" is weak or absent in these. As those objects are similar to the low- $\dot{m}$ objects identified here, it is reasonable to expect that the SEDs are also similar, i.e., that the "big blue bump" is absent and the objects have a predominantly nonthermal spectrum, as expected in the analogy to blackhole binaries. However, Satyapal, Sambruna, \& Dudik (2004) argue that the lack of an observable "big blue bump" is better explained by absorption (this possibility had also been discussed by Ho 1999). In particular, Satyapal et al. (2004) claim that the observed infrared emission lines and X-ray luminosities argue in favor of the absorption hypothesis. They furthermore cite results of detailed photoionization modeling which favor a blackbody ionizing continuum. Thus, the evidence for an intrinsic absence of a "big blue bump" in low-luminosity AGN as a class is inconclusive at present.

The spectral energy distributions (SEDs) of two of the low- $\dot{m}$ objects identified here have been analyzed in detail: Lasota et al. (1996) fitted the SED of NGC 4258 with an ADAF spectrum; however, Falcke \& Biermann (1999) argue that the SED is equally well explained by jet emission and an ADAF is not necessary, while Yuan et al. (2002) argue on the basis of more recent infrared data that a jet dominates the emission, while an ADAF still contributes. Anderson, Ulvestad, \& Ho (2004) present a similar juxtaposition of ADAF and jet models for the radio emission in six other low-luminosity AGN, which have a spectral shape similar to ADAF models but too high a radio luminosity. There is a similar controversy for NGC 1052, for which Guainazzi et al. (2000) propose an ADAF model, while more recent Chandra observations presented by Kadler et al. (2004) support a jet model. Regarding the shape of the optical continuum in this object, Keel \& Miller (1983) claim that the narrow emission-line ratios are best explained with a simple power-law ionizing spectrum, while Pequignot (1984) argues that a black-body spectrum with a higher temperature than is usual in AGN may also account for the observed line ratios. In other words, the same emissionline ratios can be accounted for by ionizing spectra both with and without a thermal component. A similar result 
is reported by Martins, Viegas, \& Gruenwald (2003) who show that different assumptions about the homogeneity of the narrow-line region can change conclusions from photoionization modeling about the shape of the ionizing continuum drastically. Again, the evidence for the absence of a "big blue bump" is inconclusive. More detailed multiwavelength observations and comparison to photoionization models are necessary.

Ho \& Peng (2001) compute radio-to-optical ratios for low-luminosity AGN using only optical emission from the nucleus itself (the larger apertures used in earlier measurements included a large contribution from starlight). The resulting radio-to-optical ratios are extremely large (up to $10^{4}$ ), similar to or even exceeding those of radio galaxies and quasars with powerful jets. This suggests that radio jets are common in low-luminosity AGN, as predicted by the analogy with black-hole binaries. However, while this analogy seems to predict both an SED without strong thermal emission and the presence of jets, observations such as those by Anderson et al. (2004) and Kadler et al. (2004) seem to imply that these are mutually exclusive when attempting to account for the radio or X-ray emission of low-luminosity AGN.

Thus, it is at present unclear whether low-luminosity AGN such as the low-accretion rate objects identified in Fig. 1 actually are scaled versions of black-hole binaries in the steady-jet, hard X-ray state. The best evidence in favor of this hypothesis seem to be the common scaling relations between X-ray binaries in the steady-jet, hard Xray state and AGN accreting at low Eddington ratios, as reported by Merloni, Heinz, \& di Matteo (2003) and Falcke, Körding, \& Markoff (2004). However, the same scaling relation seems to extend to objects at larger Eddington ratios as well, only with larger scatter; see Fig. 7 in Merloni et al. (2003). Even a rebinned version of that figure, Fig. 2 in Maccarone et al. (2003), shows steep powerlaw (or very high state) BHXBs and FR II radio galaxies falling on the same relation as the low-efficiency sources. It is somewhat surprising that the scaling does not break down altogether for the presumed high-efficiency and super-Eddington sources sources, given that the innermost regions of the accretion disks are supposed to be in entirely different physical states in those objects.

\subsection{Spectral evidence for efficient accretion?}

As a corollary to the situation for low-efficiency accretors, AGN in the high-efficiency state should be analogues of black-hole binaries in the thermal-dominant state. When an object enters the thermal-dominant state, the radio emission associated with the steady jet is quenched (Tananbaum et al. 1972), indicating that there is a much weaker jet, or no jet at all, in this state (Gallo et al. 2003). Maccarone et al. (2003) show that the radio luminosity of AGN with Eddington ratios placing them in the thermal dominant state drops below the correlation found for sources in the hard state (the correlation is based on a binned version of the data from Merloni et al. 2003 and similar to that found by Falcke et al. 2004), which is evidence in favor of such an analogy. A similar suggestion has been made by Ghisellini \& Celotti (2001), who show that the line separating low-power FR I and high-power FR II sources in a plot of AGN radio luminosity against host galaxy optical luminosity can be interpreted as a threshold in accretion rate separating the two populations. However, Cao \& Rawlings (2004) show that at least one-third of the FR I sources in the $3 \mathrm{C}$ catalog must be accreting in the radiatively efficient regime to account for their radio luminosities, if their jets are to be powered by the Blandford-Znajek mechanism. As mentioned above, they ascribe the low optical core flux of these sources to absorption.

We can assess whether the presence of jets is restricted to objects in the inefficient regime by considering Figure 2 again. What matters to the analogy between blackhole binaries and AGN is that all objects plotted in Figure 2 show extended radio emission powered by jets. In the BHXB-AGN unification picture, none of the objects with $\dot{m}>\dot{m}_{\text {crit }}$ ought to show steady jets. Of the radio-loud quasars and radio galaxies from the $3 \mathrm{C}$ survey, at least the radio-loud quasars are obviously not in the inefficiently accreting regime in Figure 2. These objects are the most luminous radio sources in the universe. As jet kinetic power correlates nearly linearly with lowfrequency radio power (Willott et al. 1999), these sources also have the most powerful jets in the universe. This is inconsistent with the expectation that the jets should be quenched (even though the quenching appears to be less extreme in AGN than in BHXB, the AGN quenching may have been underestimated due to systematic errors in luminosity and black hole measurements; Maccarone et al. 2003). I now discuss a possible resolution of this contradiction.

\subsection{Are some radio-loud AGN in a "steep power-law" state?}

Disks in efficiently accreting AGN do appear to be able to launch relatively more powerful jets than BHXBs with identical $\dot{m}$, apparently implying that BHXB-AGN unification is not perfect. However, the suggestion has been made that the radio galaxies and quasars with powerful jets are analogues of black-hole binaries in the steep power-law state with transient X-ray and radio flares, interpreted as discrete ejections of high-velocity jets (Gallo et al. 2003; Meier 2001). The observations that have most specifically been taken as evidence for this hypothesis are dips in the X-ray emission from the blazar 3C 120 linked to the appearance of new radio knots in its jet (Marscher et al. 2002). A naive scaling of the duration of these outburst in stellar-mass systems (few days) by a factor of the black hole mass ratio for AGN $\left(10^{8}-10^{10}\right)$ results in expected jet lifetimes of order $10^{6} \mathrm{y}-10^{8} \mathrm{y}$ for these AGN. It is encouraging that this brackets the typical jet age of powerful radio sources of $\approx 10^{7} \mathrm{y}$ (Willott et al. 1999). However, all the radio-loud quasars in Figure 2 have $\dot{m} \approx 0.1$, i.e., in the standard thin-disk jetless regime. Alternatively, if the accretion rates of the radio-loud quasars are systematically underestimated by a factor of $2-3$, these sources may be in the regime of the steep power-law state (indeed, the black hole masses of some of the high-redshift 3CR radio-loud quasars may have been underestimated by a comparable factor, see the note at the end of $\S$ A.1). But even if the accretion rates derived for the radio-loud quasars are not correct, there are other sources with powerful jets, but implied accretion rates firmly in the standard-disk regime. This suggests that additional factors besides the accretion rate govern the disk structure and the appearance of jets (as proposed by Meier 2001; also compare Casse \& Ferreira 
2000; Livio, Pringle, \& King 2003). This is further supported by the detection of extended radio emission with FR I morphology around the quasar E1821+643, which is optically luminous but has very low radio luminosity (Blundell \& Rawlings 2001). Conventional wisdom has it that optically luminous quasars either have FR II jets (if they are "radio loud" by either criterion for radio loudness), or a jet that is so weak that it cannot leave the host galaxy (or no jet at all). While this particular object may have FR I morphology because of strong jet precession (Blundell \& Rawlings 2001), it is possible that there are many more optically luminous quasars whose extended emission has not been detected because of a lack of sufficiently deep radio observations.

The "fast ejection" scenario for jets in powerful sources makes testable predictions about the X-ray spectra of FR I and FR II radio sources. Radio-loud AGN typically have a harder X-ray spectrum than AGN with similar optical, but lower radio luminosity, i.e., weaker or absent jets (Elvis et al. 1994). This matches the spectrum of BHXBs showing high-speed ejections in the very high state, whose distinctive feature is in fact a steep $X$ ray power-law (SPL; see McClintock \& Remillard 2004), i.e., an X-ray spectrum that is softer than that in the jet/hard state (but still harder than in the thermal dominant state). As these ejections are non-steady, there are a large number of BHXBs in the SPL state but without strong radio emission. The unification scheme thus predicts the presence of AGN with hard X-ray spectra similar to those of radio-loud quasars, but without powerful jets.

There is a possible exception to the radio-loud AGN unification scheme with implications for BHXB-AGN unification. Eracleous \& Halpern (2003) argue that broad-line radio galaxies (BLRGs) with double-peaked emission lines accrete in the low-efficiency regime. Their optical luminosity would then be lower compared to quasars with similar radio luminosity not because of obscuration, but because of a flow with lower radiative efficiency. In this case, the radio galaxies with doublepeaked lines are relatives of jet/hard-state BHXBs with steady, low-speed jets, not of their fellow FR II radio sources with optical quasar spectra and presumed highspeed non-steady ejections (jets). Indeed, BLRGs with double-peaked lines have narrow-line ratios indicating lower ionization states than is typical for radio-loud objects.

This separation into high- and low-ionization jet sources matches the findings by Willott et al. (2001) who show that is is necessary to invoke a dual-population scheme for radio galaxies in order to fit the evolution of the radio luminosity function. The high-luminosity population includes all FR II sources with strong emission lines, while the low-luminosity population includes all FR I and those FR II sources with weak emission lines, and presumably those BLRG with double-peaked emission lines. Based on such dual-population schemes, Meier (2001) had already proposed that there are two subclasses of jetted AGN with different modes of accretion and jet generation. Thus, both the double-peaked BLRGs and the dual-population scheme represent independent evidence for a class of FR II radio galaxies with different nuclear properties that can be explained by lower-efficiency accretion. It is important, though, that the luminosity separating the two radio populations with different evolution is about a factor of 10 higher than the luminosity separating sources with FR I and FR II morphology, i.e., this separation is not the one considered by Ghisellini \& Celotti (2001).

If the BHXB-AGN analogy holds up, there should be detectable differences between the jets in the high- and low-luminosity radio populations in terms of speeds and lifetimes. In this context, the study of radio galaxies with two sets of radio lobes (double-double radio galaxies) may become important. These objects are interpreted as AGN whose activity has been interrupted for a few Myr (Schoenmakers et al. 2000) and their study may provide insights into jet triggering mechanisms.

Another prediction of the BHXB-AGN unification including the third accretion sate is that there should be AGN equivalents of BHXBs in the SPL state that do not currently produce ejections, i.e., AGN which are identical to FR II radio galaxies and quasars except for emission that can be ascribed to jets, but which are different from jetless AGN in the thermal-dominant state. These issues deserve further investigation that is, however, beyond the scope of the present work.

\section{SUMMARY AND CONCLUSION}

Evidence is accumulating now that the different accretion states observed in black-hole binaries (McClintock \& Remillard 2004) will analogously occur in accretion systems in active galactic nuclei, i.e, for a unification of black-hole binaries (BHXB) and active galactic nuclei (AGN) (Meier 2001; Maccarone et al. 2003). In this paper, I present a simple observational test for the existence in AGN of the transition between a radiatively inefficient state and an efficient one thought to occur at a critical Eddington-scaled accretion rate $\dot{m}_{\text {crit }} \approx 0.01$ in BHXBs (Maccarone 2003). The test is based on the determining the location of objects in the $\left(M_{\mathrm{BH}}, L_{\mathrm{bol}}\right)$ plane, and hence their Eddington-scaled accretion rate. The underlying distribution of black hole masses and physical accretion rates is expected to vary smoothly. Therefore, a change in the accretion efficiency implies that the distribution of objects in the $\left(M_{\mathrm{BH}}, L_{\mathrm{bol}}\right)$ plane should also change at the critical accretion rate. I consider whether such a change is detectable in a sample of AGN with known bolometric luminosities and black hole masses obtained from Woo \& Urry (2002) and McLure \& Dunlop (2004). I obtain the following results:

1. The bulk of objects considered here lie in the radiatively efficient regime $\dot{m}>\dot{m}_{\text {crit }}=0.01$ of the $\left(M_{\mathrm{BH}}, L_{\mathrm{bol}}\right)$ plane (Figure 1$)$. Even though the lack of objects at $\dot{m}<\dot{m}_{\text {crit }}$ with $M_{\mathrm{BH}}>10^{9} M_{\odot}$ is mainly due to selection effects, the small number of objects with $M_{\mathrm{BH}}<10^{9} M_{\odot}$ and $\dot{m}<\dot{m}_{\text {crit }}$ is weak suggestive evidence that the density of objects decreases below the line $\dot{m}=\dot{m}_{\text {crit }}$.

2. Selection effects are important in shaping the observed distribution of objects in the $\left(M_{\mathrm{BH}}, L_{\mathrm{bol}}\right)$ plane, in particular those selection effects arising in flux-limited samples (§3.5). The most severe bias is the lack of low-luminosity objects at high redshifts from existing black hole mass surveys. These objects outnumber luminous AGN at all redshifts 
and likely have a different distribution of accretion rates than low-luminosity objects in the local universe. In particular, the apparent bimodality of the accretion rate distribution of AGN with powerful jets (Marchesini et al. 2004) is predominantly due to this selection against low-luminosity high-redshift AGN. The bimodality is strongly reduced if high-accretion rate objects are restricted to lie at $z<0.29$, the maximum redshift of the low-accretion rate objects in the Marchesini et al. (2004) sample.

3. Without a reliable method to determine an individual black hole's spin and hence the fraction $\eta$ of binding energy liberated by accretion, all determinations of accretion rates may be uncertain by a factor of three or greater (from $\eta=0.42$ for maximally co-rotating black holes to $\eta=0.06$ for non-rotating black holes, or even less for counterrotating black holes; see §3.2). This and similar uncertainties in the determination of black hole masses and bolometric luminosities mainly blur the expected change in object density at $\dot{m}=\dot{m}_{\text {crit }}$ ( $\S$ A.1, A.2). The systematic effects associated with the use of a single-band flux and a bolometric correction factor are potentially more seriously distorting the apparent distribution of objects, calling for multiwavelength SEDs to be determined for a larger sample of AGN.

4. The objects identified as low-efficiency accretors in Figure 1 are all low-luminosity AGN (§4.2). The evidence for jets and against the presence of thermal "big blue bump" emission from these objects is inconclusive on close scrutiny, although lowluminosity AGN have radio-to-optical ratios similar to those of powerful radio galaxies and radioloud quasars with jets if only their nuclear fluxes are considered (Ho \& Peng 2001). Jet and ADAF models are often pitted against each other in the literature to explain the radio and X-ray emission from low-luminosity AGN, while the unification would predict a simultaneous contribution from both (as obtained by some authors, see Ulvestad \& Ho 2001; Yuan et al. 2002, e.g.). Furthermore, photoionization models can account for the narrowline emission from these objects by an ionizing continuum both with and without a thermal component (Martins et al. 2003). Hence, the apparent lack of a big blue bump does not necessarily imply the absence of thermal emission from the accretion disk, but could also be due to absorption. On the other hand, broad-line radio galaxies with doublepeaked emission lines may be a class of objects that is genuinely in the low-efficiency regime (Eracleous \& Halpern 2003).

5. The energy output of some or all low-efficiency BHXBs is dominated by the kinetic energy of jets, so that observational tests for an equivalent kinetic energy output from low-luminosity AGN should be a powerful test of BHXB-AGN unification, e.g., by looking for evidence for deposition of the jet's kinetic energy in the AGN's surroundings.
6. Many objects in the $3 \mathrm{C}$ sample have both the most powerful radio known radio jets and accretion rates placing them firmly in the radiatively efficient regime (Figure 2 and $\S 4.3$ ), where jet production should have been quenched according to the BHXB-AGN unification scheme (as noted by Meier 2001). These objects might be the counterparts of BHXBs in the steep power-law (SPL) state with non-steady fast jets (as suggested for 3C 120; Marscher et al. 2002). Double-double radio galaxies, in which the jets have been interrupted for a few Myr (Schoenmakers et al. 2000), perhaps constitute further evidence for such an analogy. In these objects, jet production is obviously triggered by factors other than the accretion rate. Similarly, there should be AGN counterparts of SPL BHXBs which are not currently undergoing ejections.

A more accurate determination of the distribution of objects in the $\left(M_{\mathrm{BH}}, L_{\mathrm{bol}}\right)$ plane clearly depends on more accurate black hole mass measurements. The ideal sample to avoid selection effects would be a volume-limited AGN catalog with complete black hole mass identification. This would allow a determination of the accretion rate distribution in a manner similar to the computation of luminosity functions (determining volume densities instead of counting incidences). Modern surveys like the Sloan Digital Sky Survey's spectroscopic quasar survey find quasars with a much broader range in optical colors, i.e., optical SED shapes, than the Bright Quasar Survey (Schmidt \& Green 1983). Therefore, more multiwavelength SEDs are needed to obtain an accurate measurement of $L_{\text {bol }}$ for SDSS quasars, removing the substantial uncertainty associated with extrapolation from optical to bolometric luminosity. Since the X-ray emission is particularly important to test the spectral equivalence of AGN and BHXB accretion states, a sample of normal AGN with rest-frame hard X-ray spectra, deep VLA imaging to search for extended radio emission like that detected around E1821+643 (Blundell \& Rawlings 2001), and well-determined black hole masses would be particularly valuable to confirm that quasars are the counterparts of jetless BHXBs in the thermal dominant state. Clearly, the simplified theoretical treatment in $\S 2$ should be refined by using more detailed relations between the radiated flux in different parts of the spectrum and $\dot{m}$ for different accretion scenarios. In particular, this will allow to explore possible relations between radio loudness and accretion mode (see Maccarone et al. 2003 and Wang, Ho, \& Staubert 2003). In a broader context, the observed joint distribution of luminosities and black hole masses can be related to a plausible underlying distribution of accretion rates and resulting radiative efficiencies for specific models of accretion, to test their applicability to the AGN population as a whole (Merloni 2004).

This work was supported by the U.S. Department of Energy under contract No. DE-AC02-76CH03000. I am grateful to Ross McLure for providing the luminosity and black hole mass measurements from McLure \& Dunlop (2004) in electronic form, to Arieh Königl for a critical reading of the manuscript, and to Sebastian Heinz for a useful discussion. I am particularly grateful to the anonymous referee for providing rapid reviews which have led 
to substantial improvements in the paper.

Funding for the Sloan Digital Sky Survey (SDSS) has been provided by the Alfred P. Sloan Foundation, the Participating Institutions, the National Aeronautics and Space Administration, the National Science Foundation, the U.S. Department of Energy, the Japanese Monbukagakusho, and the Max Planck Society. ${ }^{1}$

The SDSS is managed by the Astrophysical Research Consortium (ARC) for the Participating Institutions. The Participating Institutions are The University of
Chicago, Fermilab, the Institute for Advanced Study, the Japan Participation Group, The Johns Hopkins University, the Korean Scientist Group, Los Alamos National Laboratory, the Max-Planck-Institute for Astronomy (MPIA), the Max-Planck-Institute for Astrophysics (MPA), New Mexico State University, University of Pittsburgh, University of Portsmouth, Princeton University, the United States Naval Observatory, and the University of Washington.

1 The SDSS Web site is http://www.sdss.org/.

\section{APPENDIX \\ SYSTEMATIC ERRORS IN THE DETERMINATION OF BLACK HOLE MASS AND ACCRETION LUMINOSITY}

\section{Errors in black hole mass and bolometric luminosity}

The bulk of the objects in Fig. 1 have virial black hole masses obtained from measurements of the width of broad lines. The largest systematic error for these comes from the uncertain orbital geometry of the broad line region emitters. McLure \& Dunlop (2004) prefer to assume a disk geometry for the BLR, which increases black hole masses by a factor of 3 compared to assuming random orbits. Different assumptions can increase the resulting $M_{\mathrm{BH}}$ by another factor of up to 150 (Krolik 2001). A global error in the orbital geometry would move the entire set of points with masses from reverberation mapping or the BLR size-luminosity relation horizontally in Fig. 1. Thus, if all other observables were known to arbitrary precision, the assumed orbital geometry would change the value of $\dot{m}_{\text {crit }}$ inferred from a plot such as Fig. 1. If the orbital geometry varies from object to object (but without systematics with respect to black hole mass or luminosity), this will introduce scatter into the determined black hole masses. Like other sources of scatter discussed below, this would lead to a smoother transition of the object density above and below $\dot{m}=\dot{m}$ crit and thus make the threshold harder to detect.

In addition to a velocity measurement, the virial mass determination requires knowledge of the size of the BLR. McLure \& Dunlop (2004) obtain both the size of the BLR and the bolometric luminosity from the same measurement of an object's continuum luminosity at either $3000 \AA$ or $5100 \AA$, using a bolometric correction of 9.8 and 5.9 , respectively, determined from an average quasar spectral energy distribution (SED) from Elvis et al. (1994). This implies a correlation between the bolometric luminosity and the black hole mass in the sense of the line in the upper left-hand corner of Fig. 1. Objects move along this line if there is an error in the determination of the optical luminosity. Since the line is nearly parallel to lines of constant $\dot{m}$, random errors in this luminosity determination do not affect the detectability of a threshold in $\dot{m}$ much.

But the use of a constant bolometric correction factor could be a source of black hole mass-dependent errors in the inferred luminosity because the optical emission comes from the "big blue bump", which is ascribed to thermal emission from the accretion disk (Shields 1978). It therefore has a temperature and hence a spectral shape that varies with black hole mass, introducing a systematic error in the luminosity. It is also relevant that the SDSS quasar survey has very different selection criteria from previous optical surveys, and may therefore include objects with SEDs different from the average SED determined from earlier quasar samples. Thus, there is likely some scatter of the true bolometric correction factor from object to object. Either change in the correction factor influences the bolometric luminosity, while the black hole mass remains unaffected. This moves objects vertically in Fig. 1 . The random scatter would blur the transition region in the same way as the scatter in the orbital geometry discussed above. A systematic error with black hole mass would shear the distribution of objects. This would still leave the threshold visible, but would lead to a threshold line that is rotated or shifted with respect to the line $\dot{m}=\dot{m}_{\text {crit }}=$ const. expected here. Thus, it would probably not affect the conclusion about the presence of a threshold, but its interpretation.

There is the additional possibility, even prediction, that the SEDs of AGN in the presumed two accretion states differ, just as the states of X-ray binaries are differentiated by their SEDs. It is thus likely that the bolometric correction factor differs between the radiatively efficient and inefficient objects, so that the calculation of the bolometric luminosity from the optical continuum luminosity would require prior knowledge of the accretion state and could not be used to determine the accretion state. The color selection algorithm for the SDSS quasars does not make any assumptions about the SED of quasars, but is designed to select all objects that are outliers from the stellar locus in color space. It is therefore in principle possible that it selects AGN in the radiatively inefficient state, as long as they pass the flux limit. However, a principal component analysis of the spectra of SDSS quasars shows that the average spectrum (the highest-order principal component) does include the big blue bump (Yip et al. 2004a,b). The variation from this average spectrum is dominated by differences in the host galaxy contribution and the slope of the power law describing the blue part of the spectrum. Thus, the vast majority of SDSS quasars must have a big blue bump, and the use of a single correction factor is in this respect adequate for this sample.

The objects from Woo \& Urry (2002) with black hole masses determined by other methods are not affected by the systematic problems discussed so far, but have some intrinsic scatter. As the various methods often cannot be crosscalibrated, it is difficult to obtain an accurate determination of the intrinsic scatter of these methods. The dispersion 
in black hole mass is usually estimated in the range 0.3 dex for masses from the $M_{\mathrm{BH}^{-}} \sigma$ relation up to 0.5 dex for the virial methods. The effect of this scatter is again to blur the transition between objects in the two accretion states.

Marchesini et al. (2004) determined black hole masses for all objects from the scaling relation between the bulge luminosity of the host galaxy and the black hole mass. They quote an uncertainty of $42 \%$ for these masses, with the same consequences of blurring the transition as for the other $M_{\mathrm{BH}}$ uncertainties. There may also be systematic errors for the high-redshift objects because the stellar populations of the high-redshift galaxies hosting the 3C AGN are different from those of the low-redshift galaxies for which the scaling relations were derived (Jong-Hak Woo, priv. comm.). These would make the black hole masses of the high-redshift objects overestimates by about a factor of 0.3-1 dex. This would also explain the absence of objects at $\dot{m}>0.3$ from Fig. 2 .

\section{Measures for accretion luminosity}

The change in the distribution of objects in the $\left(M_{\mathrm{BH}}, L_{\mathrm{bol}}\right)$ plane could be undetectable in Fig. 1 if the bolometric luminosity is not a good measure for the accretion luminosity, so that the actual accretion state is not in fact revealed by the bolometric luminosity. One problem is clearly that optical, ultraviolet, and soft X-ray emission is subject to absorption by dust close to the active nucleus, and the amount of absorption most likely depends on the viewing angle of the accretion disk (this orientation-dependent obscuration forms the basis of unified schemes for AGN; see Urry \& Padovani 1995). Therefore, it might be more appropriate to use a measure of luminosity that does not suffer from obscuration to determine the emitted bolometric luminosity, instead of extrapolating the received flux to the full solid angle by ignoring the obscuration altogether.

The regions least affected by absorption are hard X-rays and the mid- and far-infrared. Hard X-ray and midinfrared fluxes (as observed by the ISO and IRAS missions) of AGN correlate, albeit with a scatter of up to one order of magnitude ascribed to non-AGN contributions to the mid-IR flux (Lutz et al. 2004), although the bolometric luminosity is proportionally less affected. Similarly, the fraction of luminosity emitted in the far-infrared varies strongly as function of dust mass. Therefore the hard X-ray luminosity would seem most appropriate.

Indeed, often the X-ray luminosity alone is used as a measure of accretion luminosity, because the X-ray emission is believed to arise in the innermost part of the accretion system. However, the scaling of the disk temperature with black hole mass implies that the thermal emission from black-hole binaries peaks in the X-rays, while the thermal emission from AGN disks peaks in the optical/UV (the "big blue bump"). On the other hand, the observed variability in both wavelength regions implies that the optical/UV emission in AGN is reprocessed X-ray emission (Ulrich, Maraschi, \& Urry 1997; Koratkar \& Blaes 1999), or that the X-rays are comptonized optical/UV photons (Uttley et al. 2000; Chiang et al. 2000). Either way, X-ray and optical/UV emission are correlated and thus measure the same accretion luminosity. However, even bolometric corrections from the X-rays can vary by a factor of a few (Risaliti \& Elvis 2004 ). Thus, the AGN flux in any wavelength region is a measure of the accretion luminosity, but with varying amount of scatter. Additionally, if the shape of the AGN changes with the accretion state, the bolometric correction cannot be known in advance, as discussed above. To minimize the impact of these uncertainties associated with extrapolating the luminosity in a single waveband, summing the observed infrared, optical/UV and X-ray luminosity is strongly preferred, and a true bolometric luminosity is the preferred approximation to the accretion luminosity.

The radio luminosity always makes a small contribution to an object's bolometric luminosity and can safely be neglected. However, in those AGN with strong jets, the kinetic power of the jet also needs to be provided by the accretion mechanism and should therefore be included in the energy budget. This is particularly important since jets and radio lobes radiate only a small fraction of the jet's kinetic power. Therefore, the accretion luminosity should ideally be determined as the sum of the radiative bolometric luminosity of the disk system and the kinetic power of the jets. But Willott et al. (1999) concluded that the fraction of total accretion energy carried away by the jets of powerful radio galaxies and quasars ranges from only 5\%-50\%. Hence, the inclusion of jet kinetic power would not change the total power output of these objects significantly from the radiative $L_{\text {bol }}$ in Fig. 1 , although the accretion rate would be underestimated slightly, but systematically. In any case, most objects in Fig. 1 are already in the high-efficiency regime by virtue of their radiative luminosity, so their classification would not change by inclusion of their jets' kinetic power.

The situation is more complicated for objects in the low-efficiency regime. The energy output for some, if not all, X-ray binaries in the hard (low-efficiency) state is dominated by kinetic energy channeled into the jets (Fender et al. 2003; Gallo, Fender, \& Pooley 2003; Malzac, Merloni, \& Fabian 2004). The radiative luminosity will then be only a small fraction of the accretion luminosity. However, as long as the overall scaling of the radiative efficiency with accretion rate is not different from what is assumed here (Equation 4) - in other words, as long as the jet is powered by accretion energy that would otherwise be advected into the black hole - , the accretion rate as inferred from the radiative luminosity will still be correct. Assuming a complete analogy between black-hole binary and AGN accretion systems has further interesting implications for the presence or absence of jets in AGN as a function of accretion state, which is discussed in $\S 4.3$.

In summary, the radiative bolometric luminosity is the adequate measure of total accretion luminosity. To avoid the uncertainties associated with using a single bolometric correction factor, it would be desirable to obtain true multiwavelength SEDs for a representative sample of SDSS quasars.

\section{REFERENCES}

Abramowicz, M. A. 2004, in Growing Black Holes: Accretion in a Cosmological Context, Garching 2004, ed. A. Merloni, S. Nayakshin, \& R. Sunyaev, ESO Astrophysics Symposia

(Berlin: Springer), in press [astro-ph/0411185]
Anderson, J. M., Ulvestad, J. S., \& Ho, L. C. 2004, ApJ, 603, 42 
Barret, D., \& Olive, J. 2002, ApJ, 576, 391

Begelman, M. C., \& Meier, D. L. 1982, ApJ, 253, 873

Blandford, R. D., \& Begelman, M. C. 1999, MNRAS, 303, L1

Blundell, K. M., \& Rawlings, S. 2001, ApJ, 562, L5

Cao, X., \& Rawlings, S. 2004, MNRAS, 349, 1419

Casse, F., \& Ferreira, J. 2000, A\&A, 361, 1178

Chen, X., Abramowicz, M. A., Lasota, J., Narayan, R., \& Yi, I. 1995, ApJ, 443, L61

Chiaberge, M., Macchetto, F. D., Sparks, W. B., Capetti, A., Allen, M. G., \& Martel, A. R. 2002, ApJ, 571, 247

Chiang, J., Reynolds, C. S., Blaes, O. M., Nowak, M. A., Murray, N., Madejski, G., Marshall, H. L., \& Magdziarz, P. 2000, ApJ, 528, 292

Colla, G., Fanti, C., Fanti, R., Gioia, I., Lari, C., Lequeux, J., Lucas, R., \& Ulrich, M.-H. 1975, A\&A, 38, 209

Elvis, M., et al. 1994, ApJS, 95, 1

Eracleous, M., \& Halpern, J. P. 2003, ApJ, 599, 886

Esin, A. A., McClintock, J. E., \& Narayan, R. 1997, ApJ, 489, 865

Falcke, H., \& Biermann, P. L. 1999, A\&A, 342, 49

Falcke, H., Körding, E., \& Markoff, S. 2004, A\&A, 414, 895

Fender, R. P., Belloni, T. M., \& Gallo, E. 2004, MNRAS, 538

Fender, R. P., Gallo, E., \& Jonker, P. G. 2003, MNRAS, 343, L99

Gallo, E., Fender, R. P., \& Pooley, G. G. 2003, MNRAS, 344, 60

Ghisellini, G., \& Celotti, A. 2001, A\&A, 379, L1

Guainazzi, M., Oosterbroek, T., Antonelli, L. A., \& Matt, G. 2000, A\&A, 364, L80

Haardt, F., \& Maraschi, L. 1991, ApJ, 380, L51

Heckman, T. M. 1980, A\&A, 87, 152

Ho, L. C. 1999 , ApJ, 516, 672

Ho, L. C., \& Peng, C. Y. 2001, ApJ, 555, 650

Hutchings, J. B., Frenette, D., Hanisch, R., Mo, J., Dumont, P. J., Redding, D. C., \& Neff, S. G. 2002, AJ, 123, 2936

Kadler, M., Kerp, J., Ros, E., Falcke, H., Pogge, R. W., \& Zensus, J. A. 2004, A\&A, 420, 467

Keel, W. C., \& Miller, J. S. 1983, ApJ, 266, L89

Koratkar, A., \& Blaes, O. 1999, PASP, 111, 1

Krolik, J. H. 2001, ApJ, 551, 72

Laing, R. A., Riley, J. M., \& Longair, M. S. 1983, MNRAS, 204 151

Lasota, J.-P., Abramowicz, M. A., Chen, X., Krolik, J., Narayan, R., \& Yi, I. 1996, ApJ, 462, 142

Liu, F. K., \& Zhang, Y. H. 2002, A\&A, 381, 757

Livio, M., Pringle, J. E., \& King, A. R. 2003, ApJ, 593, 184

Lutz, D., Maiolino, R., Spoon, H. W. W., \& Moorwood, A. F. M. 2004, A\&A, 418, 465

Maccarone, T. J. 2003, A\&A, 409, 697

Maccarone, T. J., \& Coppi, P. S. 2003, MNRAS, 338, 189

Maccarone, T. J., Gallo, E., \& Fender, R. 2003, MNRAS, 345, L19

Malzac, J., Merloni, A., \& Fabian, A. C. 2004, MNRAS, 351, 253

Marchesini, D., Celotti, A., \& Ferrarese, L. 2004, MNRAS, 351 733

Marconi, A., \& Hunt, L. K. 2003, ApJ, 589, L21

Marscher, A. P., Jorstad, S. G., Gómez, J., Aller, M. F., Teräsranta, H., Lister, M. L., \& Stirling, A. M. 2002, Nature, 417, 625

Martins, L. P., Viegas, S. M., \& Gruenwald, R. 2003, ApJ, 587, 562

McClintock, J. E., \& Remillard, R. A. 2004, in Compact Stellar Xray Sources, ed. W. H. G. Lewin \& M. van der Klis (Cambridge University Press, in press [astro-ph/0306213])
McLure, R. J., \& Dunlop, J. S. 2001, MNRAS, 327, 199

-. 2004, MNRAS, 352, 1390

Meier, D. L. 2001, ApJ, 548, L9

Merloni, A. 2004, MNRAS, 353, 1035

Merloni, A., Heinz, S., \& di Matteo, T. 2003, MNRAS, 345, 1057

Meyer, F., Liu, B. F., \& Meyer-Hofmeister, E. 2000, A\&A, 354, L67

Nagar, N. M., Falcke, H., Wilson, A. S., \& Ulvestad, J. S. 2002, A\&A, 392, 53

Narayan, R., \& Yi, I. 1995, ApJ, 452, 710

Pequignot, D. 1984, A\&A, 131, 159

Proga, D. 2005, in Growing Black Holes: Accretion in Cosmological Context, ed. A. Merloni, S. Nayakshin, \& R. Sunyaev, ESO Astrophysics Symposia (Berlin: Springer), in press [astro$\mathrm{ph} / 0409475$

Richards, G. T., et al. 2002, AJ, 123, 2945

Risaliti, G., \& Elvis, M. 2004, in Supermassive Black Holes in the Distant Universe, ed. A. J. Barger (Kluwer Academic Publishers, in press [astro-ph/0403618])

Satyapal, S., Sambruna, R. M., \& Dudik, R. P. 2004, A\&A, 414, 825

Schmidt, M., \& Green, R. F. 1983, ApJ, 269, 352

Schneider, D. P., et al. 2003, AJ, 126, 2579

Schoenmakers, A. P., de Bruyn, A. G., Röttgering, H. J. A., van der Laan, H., \& Kaiser, C. R. 2000, MNRAS, 315, 371

Shakura, N. I., \& Sunyaev, R. A. 1973, A\&A, 24, 337

Shields, G. A. 1978, Nature, 272, 706

Spinrad, H., Marr, J., Aguilar, L., \& Djorgovski, S. 1985, PASP 97,932

Tananbaum, H., Gursky, H., Kellogg, E., Giacconi, R., \& Jones, C. 1972, ApJ, 177, L5+

Treister, E., et al. 2004, ApJ, 616, 123

Ulrich, M., Maraschi, L., \& Urry, C. M. 1997, ARA\&A, 35, 445

Ulvestad, J. S., \& Ho, L. C. 2001, ApJ, 562, L133

Urry, C. M., \& Padovani, P. 1995, PASP, 107, 803

Uttley, P., McHardy, I. M., Papadakis, I. E., Cagnoni, I., \& Fruscione, A. 2000, MNRAS, 312, 880

Vanden Berk, D. E., et al. 2001, AJ, 122, 549

Wang, J.-M., Ho, L. C., \& Staubert, R. 2003, A\&A, 409, 887

Willott, C. J., Rawlings, S., Blundell, K. M., \& Lacy, M. 1999 MNRAS, 309, 1017

Willott, C. J., Rawlings, S., Blundell, K. M., Lacy, M., \& Eales, S. A. 2001, MNRAS, 322, 536

Wolf, C., Wisotzki, L., Borch, A., Dye, S., Kleinheinrich, M., \& Meisenheimer, K. 2003, A\&A, 408, 499

Woo, J., \& Urry, C. M. 2002, ApJ, 579, 530

Yip, C., Connolly, A., Berk, D. V., Ma, Z., Frieman, J., SubbaRao, M., \& Szalay, A. 2004a, in ASP Conf. Ser.: "AGN physics with the SDSS", in press [astro-ph/0403560], ed. G. T. Richards \& P. B. Hall

Yip, C. W., et al. 2004b, AJ, 128, 2603

Yuan, F., Markoff, S., Falcke, H., \& Biermann, P. L. 2002, A\&A 391, 139 